\title{
ENHANCING A DRIVER'S SITUATION AWARENESS USING A GLOBAL VIEW MAP
}

\author{
Hong Cheng ${ }^{1,2}$, Zicheng Liu ${ }^{3}$, Nanning Zheng ${ }^{2}$, Jie Yang ${ }^{1}$ \\ ${ }^{1}$ School of Computer Science, Carnegie Mellon University, USA \\ ${ }^{2}$ Institute of Artificial Intelligence and Robotics, Xi' an Jiaotong University, China \\ ${ }^{3}$ Microsoft Research, One Microsoft Way, Redmond, WA98052,USA \\ Email:\{hongc, jie.yang\}@cs.cmu.edu, zliu@microsoft.com, nnzheng@mail.xjtu.edu.cn
}

\begin{abstract}
This paper proposes a novel method to enhance a driver's situation awareness by dynamically providing a global view of surroundings for the driver. The surroundings of a vehicle are captured by an omni-directional vision system mounted on the top of the vehicle. The video stream from the camera is processed to detect nearby vehicles. Positions of these detected objects are overlaid on a global view of a local map (e.g., an aerial imagery or satellite imagery map). We establish the relationship between the omni-directional vision system and the global view map. The global view map dynamically provides a realistic perspective view of the driving environment. This map can be projected onto an HUD on the windshield. By looking at the display, a driver can have a global picture of the situation and potentially produce a good driving strategy. We illustrate the proposed method by dynamically mapping a video stream onto Google Earth map.
\end{abstract}

\section{INTRODUCTION}

Driver inattention is a major contributor to highway crashes. The National Highway Traffic Safety Administration estimates that at least $25 \%$ of police-reported crashes involve some form of driver inattention [1]. Driving is a process that requires a driver to distribute his/her attention among different subtasks. First of all, a driver needs to pay attention to issues directly related to safety, including the surrounding traffic, dashboard displays, and other influx of information on the road such as traffic lights and road signs. In addition, the driver may choose to talk to passenger, listen to the radio, and talk on the cell phone. Therefore, situation awareness plays an important role in driving safety. In this research, we are developing technologies to provide a driver with the information of dynamic surroundings around the vehicle that he/she is driving to enhance his/her situation awareness.

Situation awareness is defined as the perception of the elements in the environment within a volume of time and space, the comprehension of their meaning, and the projection of their status in the near future [2]. Sensing and representing information is a key for situation awareness in driving a vehi- cle. Much research has been directed to improving in-vehicle information presentation. Green et al. surveyed early studies on human factor tests in navigation displays [3]. They described objectives, principles and guidelines for the design of in-vehicle devices. Dale et al. investigated the problem of generating natural route descriptions for navigational assistance [4]. Lee et al. developed a situationally appropriate map system for drivers [5].

Navigation user interfaces have changed dramatically over the last few years due to the availability of electronic maps and the Global Positioning System (GPS). The displays in current GPS navigation systems show the location of a vehicle on a graphical map in a way that's similar to looking straight down at a paper map. Recently, several companies, such as Microsoft and Google, have started proving global view maps, such as aerial imagery maps, satellite imagery maps, and bird's eye view maps. For example, in the bird's eye view mode, Microsoft's Windows Live Local consists of high resolution aerial imagery taken from an angle rather than straight down from four directions. Besides the GPS, a vehicle can also obtain information about the driving environment from other sensors, such as video cameras mounted in various positions, thermal infrared imagers, RADAR, LIDAR, and ultrasonic sensors [6]. Among these sensors, video cameras are attractive from a packaging and cost perspective. Recent advances in computer vision and image processing technologies have made it possible to apply video-based sensors along with the GPS in driving assistance applications.

In this paper, we propose a novel method to enhance situation awareness by dynamically providing a global view of surrounding for drivers. The surrounding of a vehicle will be captured by an omni-directional vision system on the top of a vehicle. In order to obtain high quality of surrounding images, we use an omni-directional vision system consisting of multiple cameras [7], rather than a catadioptric camera used by the most existing system for intelligent vehicles [8]. The video stream from the camera is processed to detect nearby vehicles and obstacles. Positions of these detected objects will be overlaid on a global view map of the vehicle. We deduce the mapping between an omni-directional vision system and global 


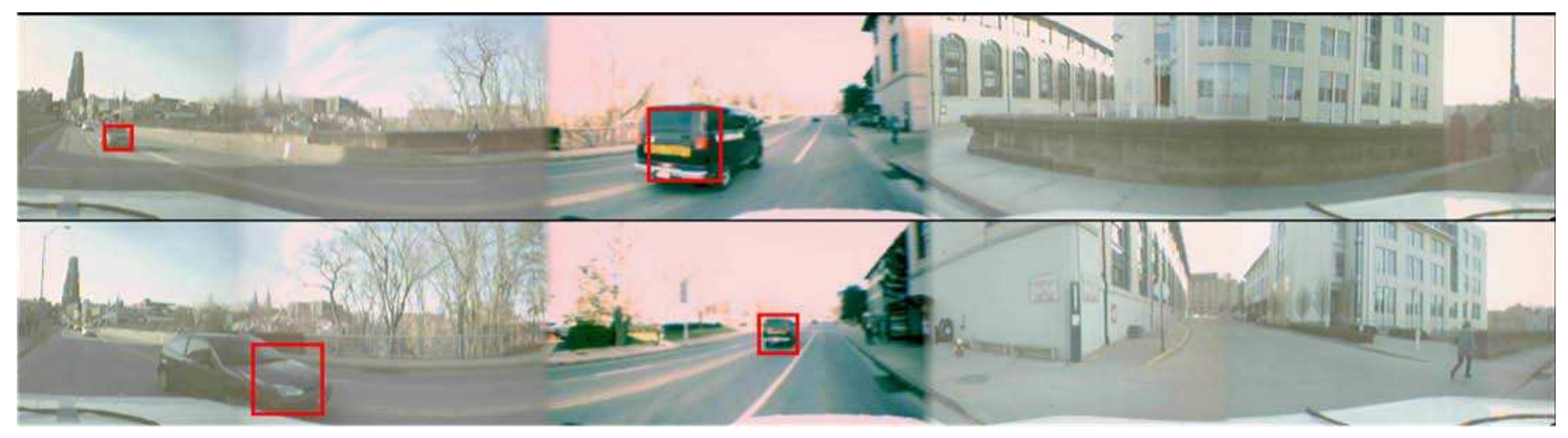

Fig. 1. Panorama Images Taken by a Camera Array Sitting on Top of a Vehicle

view map. This map can be projected onto a Head-Up Display (HUD) on the windshield and provide a dramatically realistic perspective view of the driving environment. By looking at the display, a driver can have a global picture of the situation and likely produce a good driving strategy. The rest of this paper is organized as the following: Section 2 describes the problem and proposed approach. Section 3 discusses the imaging model of our camera system. In section 4, we illustrate the proposed method by an example that maps vehicles detected from the video stream captured by omni-directional vision system onto the Google Earth map. We conclude our work in section 5 .

\section{THE PROBLEM AND PROPOSED APPROACH}

The field of view, which is the part of the observable world that is seen at any given moment, plays an important role in driving safety. While a human has an almost 180-degree forward-facing field of view, his/her binocular vision, which is important for depth perception, only covers 140 degrees of the field of vision. In a driving situation, it is desirable to have a complete 360-degree field of view. In order to expend a driver's field of view, automobile manufacturers have equipped a rear-view mirror and side mirrors on vehicles. More recently, rear-view video cameras have been added to many new model cars to enhance the ability of the rear-view mirror by showing the road directly behind the car. These camera systems are usually mounted to the bumper or lower parts of the car allowing for better rear visibility. However, looking at mirrors can move a driver's attention away from the road.

Adding sensors and devices in a vehicle can potentially lead to more distractions. Inattention is one of leading causes of car accidents, estimated to account for $25 \%$ of all road traffic accidents. Our goal, therefore, is to increase a driver's field of view without adding distraction sources. In this paper, we propose to capture surroundings of a vehicle by an omnidirectional vision system mounted on the top of a vehicle and display the dynamic global view on the windshield using an
HUD). In this way, a driver can obtain a global view of the surrounding without shifting his/her attention away from the front view of the vehicle.

Omni-directional vision system have been previously used in intelligent vehicle applications, such as vehicle tracking, indoor parking lot, and driver monitoring driver, etc [8]. These applications used different omni-directional sensors, such as wide Field-Of-View(FOV) dioptric cameras, catadioptric cameras, Pan-Title-Zoom (PTZ) cameras and polydioptric cameras. Both wide FOV dioptric cameras and catadioptric cameras have some limitations. First, their images are heavily distorted, and we have to spend much time on correcting the distortion. Second, they can't provide high resolution images of surroundings. PTZ cameras are often used in environment surveillance by moving the cameras. Although PTZ cameras can provide high resolution images, mechanical motion of the cameras causes system response slow. Instead of use these cameras, we will use an omni-directional vision system consisting of multiple cameras to capture a full view of surroundings around a vehicle with the high resolution up to $1600 \times 320$ simultaneously [7]. Fig. 1 shows examples of two panoramic images captured in our experiment. Rectangles in the images are the detected vehicles..

However, the panoramic video stream from the omnidirectional camera cannot easily be understood by a driver, so we map the driving situation onto a global view map. That is, we automatically extract objects (vehicles, pedestrians, etc) from the video stream and mark their positions on the global view map. We use a hypothesis-validation structure to detect the nearby vehicles surrounding a host vehicle [9]. Without losing generality, in this paper, we have utilized Google Earth, which can provide us with high quality and resolution aerial and satellite images including highway, street, and more. In addition, data import feature from the Google Earth makes it possible to sense and represent the dynamic information surrounding a host vehicle and import our custom geographic data into the Google Earth application. 


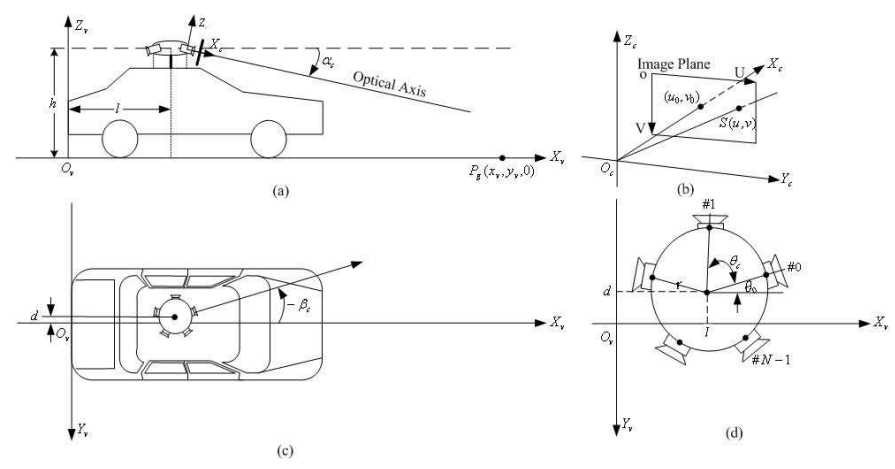

Fig. 2. Geometric Relationship among the Vehicle, Camera Array, and Image Coordinate System of each Individual Camera: (a) Front View ; (b) Image Plane of an individual camera; (c) Aerial View; (d) Camera Array Layout.

\section{THE IMAGING MODEL}

In this section, we describe the mathematical model of panoramic imaging and provide a context for the mapping between the panoramic image and the global electronic map.

There are three different coordinate systems as shown in Fig. 2. $X_{v} Y_{v} Z_{v}$ is the vehicle coordinate system. $X_{c} Y_{c} Z_{c}$ is the coordinate system for individual camera $c$ in the camera array where $c=0, \ldots, N-1$ and $N$ is the number of cameras. $U O V$ is the image coordinate system of camera $c$. Let $r$ denote the radius of the camera array, and $\theta=2 \pi / N$ the shift angle. The 3D coordinate of the camera array center is $[l, d, h]^{T}$ in the vehicle coordinate systems. The orientation of camera $c$ is defined by two rotation angles $\alpha_{c}$ and $\beta_{c}$ as shown in Fig.2 (a) and (b).

Assuming the road surface is horizontal, the coordinate of the optical center of camera $c$ in the $X_{v} Y_{v} Z_{v}$ coordinate system is

$$
T_{0}^{c}=\left[l+r \cos \beta_{c}, d+r \sin \beta_{c}, h\right]^{T}=\left[l^{\prime}, d^{\prime}, h\right]^{T} .
$$

Given any 3D point, let $P_{v}=\left[x_{v}, y_{v}, z_{v}\right]^{T}$ denote its coordinate in the vehicle coordinate system $X_{v} Y_{v} Z_{v}$. Let $P_{c}=\left[x_{c}, y_{c}, z_{c}\right]^{T}$ denote its coordinate in the camera coordinate system $X_{c} Y_{c} Z_{c}$. We have

$P_{v}=\left[\begin{array}{ccc}\cos \alpha_{c} \cos \beta_{c} & \sin \beta_{c} & -\sin \alpha_{c} \cos \beta_{c} \\ -\cos \alpha_{c} \sin \beta_{c} & \cos \beta_{c} & \sin \alpha_{c} \sin \beta_{c} \\ \sin \alpha_{c} & 0 & \cos \alpha_{c}\end{array}\right] P_{c}+T_{0}^{c}$.

Considering camera c's image plane $U O V$ as shown in Fig. 2 (b). Let $\left(u_{0}, v_{0}\right)$ denote the coordinate of the principal point. According to perspective projection, any point $S(u, v)$ on the image plane satisfies the following equation

$$
\frac{y_{c}}{x_{c}}=\frac{u-u_{0}}{f_{u}}, \quad \frac{z_{c}}{x_{c}}=-\frac{v-v_{0}}{f_{v}},
$$

where $f_{u}, f_{v}$ are the scale factor along $U$ and $V$ axis, respectively.
Let $x_{c}=t, t \in[0, \infty)$, and applying Equ. (3) yields

$$
x_{c}=t, \quad y_{c}=t \cdot \frac{u-u_{0}}{f_{u}}, \quad z_{c}=-t \cdot \frac{v-v_{0}}{f_{v}} .
$$

Therefore, the parametric equation of line $O_{c} S$ can be written as

$$
\left[x_{c}, y_{c}, z_{c}\right]^{T}=t \cdot\left[1, \frac{u-u_{0}}{f_{u}},-\frac{v-v_{0}}{f_{v}}\right]^{T} .
$$

Substituting Equ. (5) into Equ. (2), we obtain the line equation in the vehicle coordinate system

$$
\left[\begin{array}{l}
x_{v} \\
y_{v} \\
z_{v}
\end{array}\right]=t \cdot \mathbf{R} \cdot\left[\begin{array}{c}
1 \\
\frac{u-u_{0}}{f_{u}} \\
-\frac{v-v_{0}}{f_{v}}
\end{array}\right]+\left[\begin{array}{l}
l^{\prime} \\
d^{\prime} \\
h
\end{array}\right]
$$

where $\mathbf{R}$ is the rotation matrix in Equ. (2).

If we assume the road is flat, the equation of the road plane is $z_{v}=0$. Therefore the intersection of the line $O_{c} S$ with the road plane can be obtained by setting $z_{v}=0$ in Equ. (6), which yields

$$
\begin{aligned}
& x_{v}(u, v)=h^{\prime}\left[\begin{array}{lll}
u^{\prime} & v^{\prime} & 1
\end{array}\right]\left[\begin{array}{c}
\sin \beta_{c} \\
\sin \alpha_{c} \cos \beta_{c} \\
\cos \alpha_{c} \cos \beta_{c}
\end{array}\right]+l^{\prime}, \\
& y_{v}(u, v)=h^{\prime}\left[\begin{array}{lll}
u^{\prime} & v^{\prime} & 1
\end{array}\right]\left[\begin{array}{c}
\cos \beta_{c} \\
-\sin \alpha_{c} \sin \beta_{c} \\
-\cos \alpha_{c} \sin \beta_{c}
\end{array}\right]+d^{\prime}
\end{aligned}
$$

where $h^{\prime}=\frac{h}{v^{\prime} \cos \alpha_{c}-\sin \alpha_{c}}, u^{\prime}=\frac{u-u_{0}}{f_{u}}, v^{\prime}=\frac{v-v_{0}}{f_{v}}$.

Note that the object detection is performed on the stitched panoramic image. Given a pixel on the panoramic image, its corresponding positions on the individual camera image planes are obtained from the stitching table, which is generated by a stitching calibration process [7].

\section{COMBINING PANORAMIC IMAGES WITH ELECTRONIC MAPS}

Electronic map services such as Microsoft Virtual Earth and Google Earth can help to reduce a driver's load by providing high quality electronic route and turn-to-turn directions. For example, Fig. 3 shows the route, generated by Google Earth, around Carnegie Mellon University.

We can further reduce a driver's cognitive load by combining the images captured by the omni-directional camera with the electronic map in real time. In particular, we perform image analysis to detect surrounding objects such as vehicles and pedestrians, and display the detected objects on the electronic map.

In this paper, we mainly focus on vehicle detection. Our vehicle detection approach includes two basic phases. In the hypothesis generation phase, we first determine the Regions 


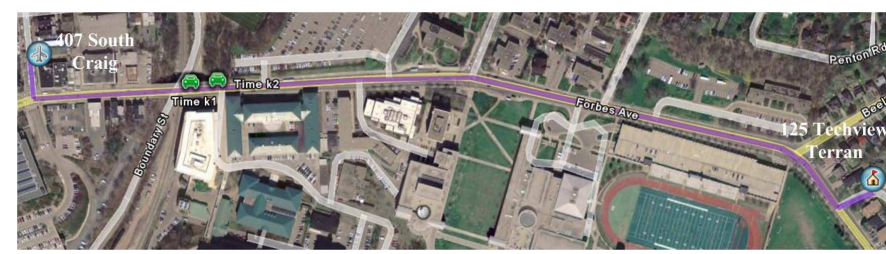

Fig. 3. Google Global Navigation Map

of Interest (ROI) in an image according to lane vanishing points. From the analysis of horizontal and vertical edges in the image, vehicle hypothesis lists are generated for each ROI. In the hypothesis validation phase, we have developed a vehicle validation system by using Support Vector Machine (SVM) and Gabor features. For details we refer to [9]. Fig. 1 shows the results of vehicle detection in two omnidirectional images.

Let $V_{w}=[\phi, \gamma, \eta]^{T}$ denote the coordinate of the host vehicle where $\phi$ and $\gamma$ are provided by an in-vehicle GPS device, and $\eta$ is the direction of vehicle. Let $\left(x_{v}(u, v), y_{v}(u, v)\right)$ denote the coordinate of a detected vehicle, the latitude and longitude of the detected vehicle can be written as [10]:

$$
\left[\begin{array}{c}
\phi_{o} \\
\gamma_{o}
\end{array}\right]=\left[\begin{array}{ccc}
k_{1} \cos \eta & -k_{1} \sin \eta & \phi \\
k_{2} \sin \eta & k_{2} \cos \eta & \gamma
\end{array}\right]\left[\begin{array}{c}
x_{v}(u, v) \\
y_{v}(u, v) \\
1
\end{array}\right]
$$

where $k_{1}$ and $k_{2}$ are scalar values to put the points into the earth's longitude and latitude coordinate system. Thus we can display the detected vehicle on an electronic map. Fig. 4 shows the results of displaying the detected vehicles in the two panoramic images as shown in Fig. 1 onto the Google Earth map.

\section{CONCLUSIONS}

In this paper, we proposed a novel technique to improve a driver's situation awareness by dynamically combining panoramic imaging with global maps. By displaying surrounding objects on the global map, the driver can easily see his/her surroundings without having to turn his/her head off the driving direction. We believe that this will significantly reduce the driver's load and improve driving safety.

\section{Acknowledgements}

This work was partially supported by the gift grant from the General Motors. The first author was also supported by the scholarship from China Scholarship Council.

\section{REFERENCES}

[1] J. C. Stutts, D. W. Reinfurt, L. Staplin, and E. A. Rodgman, "The role of driver distraction in traffic

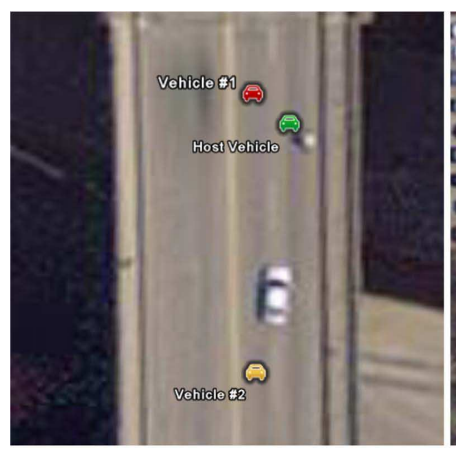

(a)

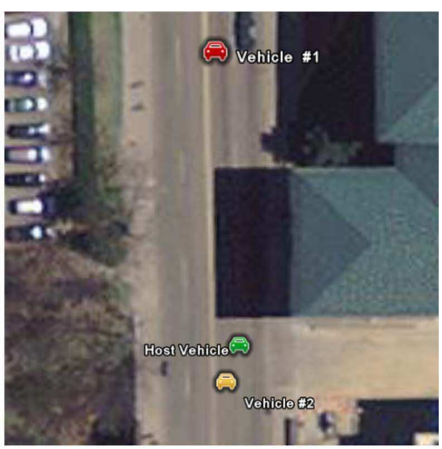

(b)
Fig. 4. Mapping from Detected Objects onto Google Earth Map: (a) The objects are detected from the top image in Fig.1; (b) The objects are detected from the bottom image in Fig.1.

crashes," Report of AAA Foundation for Traffic Safety, www.aaafoundation.org, 2001.

[2] M. R. Endsley, "Situation awareness global assessment technique (SAGAT)," Proceedings of the NAECON, pp. 789-795, 1988.

[3] P. Green, W. Levison, G. Paelke, and C. Serafin, "Preliminary human factors design guidelines for driver information systems," In UMTRI-93-21, 1995.

[4] R. Dale, S. Geldof, and J.-P. Prost, "Using natural language generation in automatic route description," Journal of Research and Practice in Information Technology, vol. 37, no. 1, 2005.

[5] J. Lee, J. Forlizzi, and S. E. Hudson, "Studying the effectiveness of move: A contextually optimized invehicle navigation system," Proceedings of ACM CHI, 2005.

[6] W.J. Fleming, "Overview of automotive sensors," IEEE Sensors Journal, vol. 1, no. 4, pp. 296-308, 2001.

[7] R. Cutler, Y. Rui, A. Gupta, JJ Cadiz, I. Tashev, L. He, A. Colburn, Z.Zhang, Z. Liu, and S. Silverberg, "Distributed meetings: A meeting capture and broadcasting system," ACM Multimedia, 2002.

[8] T. Gandhi and M. Trivedi, "Vehicle surround capture: Survey of techniques and a novel omni-video-based approach for dynamic panoramic surround maps," IEEE Transactions on ITS, vol. 7, no. 3, pp. 293- 308, 2006.

[9] H. Cheng, N. N. Zheng, C.Sun, and H. van de Wetering, "Vanishing point and gabor feature based multiresolution on-road vehicle detection," ISNN, May 2006.

[10] F. G. Gonzalez, R. Andres, and ect., "Black knight: An autonomous vehicle for competition," Journal of Robotics Systems, vol. 21, no. 9, pp. 451-460, 2004. 